\title{
Channel Measurement for Multiple Frequency Bands in Subway Tunnel Scenario
}

\author{
Zhuomin Hu $\mathbb{D}^{1},{ }^{1}$ Wenli Ji, ${ }^{2}$ Hengkai Zhao $\left(\mathbb{D},{ }^{1}\right.$ Xuping Zhai, ${ }^{1}$ Asad Saleem $\left(\mathbb{D},{ }^{3}\right.$ \\ and Guoxin Zheng ${ }^{1}$ \\ ${ }^{1}$ Key Laboratory of Specialty Fiber Optics and Optical Access Networks, \\ Joiny International Research Laboratory of Specialty Fiber Optics and Advanced Communication, Shanghai University, \\ Shanghai 200444, China \\ ${ }^{2}$ System Integration Department, Technical Center, Shanghai Shentong Metro Group Co.,Ltd, Shanghai 201103, China \\ ${ }^{3}$ Guangdong Engineering Research Center of Base Station Antennas and Propagation, \\ Key Laboratory of Antennas and Propagation, Shenzhen University, Shenzhen 518000, China
}

Correspondence should be addressed to Hengkai Zhao; zhaohk@shu.edu.cn

Received 9 March 2021; Accepted 18 May 2021; Published 3 June 2021

Academic Editor: Renato Cicchetti

Copyright (C) 2021 Zhuomin Hu et al. This is an open access article distributed under the Creative Commons Attribution License, which permits unrestricted use, distribution, and reproduction in any medium, provided the original work is properly cited.

In next-generation radio communication systems, the use of higher frequency bands and the massive multiple-input-multipleoutput (MIMO) systems has turned into hot research topics because they have the potential to increase network capacity significantly by exploiting the available narrowband and broadband spectrums. Therefore, the narrowband channel measurements are executed at the following five potential frequency bands, including $2.6 \mathrm{GHz}, 3.5 \mathrm{GHz}, 5.6 \mathrm{GHz}, 10 \mathrm{GHz}$, and $28 \mathrm{GHz}$ in the Shanghai subway tunnel environment in order to fulfill the latest standards of fifth generation (5G). Moreover, in the broadband channel measurements, the center frequency is $3.5 \mathrm{GHz}$ and $5.6 \mathrm{GHz}$ and the bandwidth is considered as $160 \mathrm{MHz}$, respectively. At the transmitter (Tx) side, a uniform rectangular antenna array composed of 32 elements is fixed on the platform near the tunnel walls. The receiver $(\mathrm{Rx})$ is equipped with a uniform cylindrical antenna array consisting of 64 elements, which is set on a trolley along the track. Based on the acquired massive MIMO channel impulse responses, delay spread, angle spread, eigenvalue and channel capacity are analyzed. The results reveal that the multipath delay in the tunnel scenario is quite short, the delay spread and angle spread drop rapidly as the distance between $\mathrm{Tx}$ and $\mathrm{Rx}$ increases and the channel matrix gradually becomes serious. This research provides a reference for the deployment of future $5 \mathrm{G}$ systems in the subway tunnel.

\section{Introduction}

In recent years, in order to ensure the safety and efficiency of train operations, it is necessary to transmit data such as ultra-high-definition video stream monitoring, train operation control, and on-board sensing at a high rate and high reliability between train and ground, which has created an urgent need for the application and deployment of 5G in subway communications. The characteristics of the wireless propagation channel determine the final performance of the wireless communication system. Therefore, it is very important to detect the actual performance of wireless propagation channels in a real tunnel environment. So far, a large number of channel measurements [1-14] have been carried out in different subway tunnel environments. The pioneering paper [1] shows that multiple reflections on the tunnel wall and changes in tunnel cross-section result in a relatively high diversity of MIMO channels in the tunnel environment. According to the modal theory [2], the tunnel can be regarded as a lossy waveguide, and the number of active modes decreases rapidly as the Tx-Rx distance increases, which limits the degree of freedom of the MIMO channel matrix. However, compared with single-inputsingle-output (SISO) channel, the improvement in channel capacity is significant. On the basis of channel measurements in a subway tunnel, the influence of polarization diversity, 
space diversity, and cross section of the tunnel on the capacity of MIMO channel capacity is considered in [3-6], which provides suggestions for the design of the MIMO system in tunnel scenario. It is pointed out that the increment of the cross-sectional area improves the MIMO channel performance through measurements conducted in a two-section tunnel-like environment in [7]. In [8-10], the first keyhole measurement was introduced in the train-toground communication system to evaluate the keyhole probability of different polarization combinations and tunnel cross sections, and it reveals that the impact of the keyhole effect is limited. Broadband measurements are conducted in the tunnel environment in [11-13]. The influence of train cars on the wireless channel in the tunnel is considered and examined in $[14,15]$. Massive MIMO channels are measured in various scenarios strongly related to the subway tunnel environments, such as subway platform halls at $6 \mathrm{GHz}$ and $11 \mathrm{GHz}[16,17]$ and intrawagon environment at the millimeter wave (mmWave) bands [18]. It is proved that sum-rate capacity increases with the increase of frequency. In $[19,20]$, the scholars divide the tunnel into different propagation zones and propose a propagation model that covers different propagation mechanisms in the tunnel. In [21], a vector parabolic equation (VPE) field prediction model is proposed, which can be used to analyze radio propagation in railway rectangular tunnels. On the basis of [21], VPE modeling has been improved and tunnel roughness is incorporated as a parameter in [22], which can be further used for various tunnel geometries with arbitrary cross sections and curvature variations. A fast and accurate radio wave propagation modeling combining the hybrid VPE and waveguide mode theory is proposed in [23]. However, to the best of the authors' knowledge, the current MIMO antenna system used for channel measurements in real tunnel scenarios does not exceed $4 \times 4$ and the research on the long-distance train-to-ground communication system in actual subway tunnel scenarios is particularly lacking. Therefore, it is difficult to provide engineering guidance for the design of $5 \mathrm{G}$ systems in tunnel environments.

In this paper, we conduct narrowband and broadband wireless communication measurements in Shanghai Metro. In the narrowband measurements, five potential frequency bands of $5 \mathrm{G}$, including $2.6 \mathrm{GHz}, 3.5 \mathrm{GHz}, 5.6 \mathrm{GHz}, 10 \mathrm{GHz}$, and $28 \mathrm{GHz}$, are selected, and the propagation characteristics of signals in the tunnel environment are investigated. In the broadband measurements, the center frequency of the carrier is $3.5 \mathrm{GHz}$ and $5.6 \mathrm{GHz}$, and the bandwidth is deliberated as $160 \mathrm{MHz}$. The $\mathrm{Tx}$ and $\mathrm{Rx}$ antenna arrays are composed of 32 and 64 elements, respectively. In order to simulate the actual wireless communications in a subway tunnel, the transmitter is fixed on the platform close to the tunnel wall, and the receiver is placed in a trolley that can move along the track. Based on the collected data, we modeled the path loss parameters and analyzed the power delay profile (PDP), root-mean-square delay spread (RMSDS), root-mean-square angle spread (RMS-AS), eigenvalue, and channel capacity.

The rest of this paper is organized as follows. In Section 2 , the environment and system for narrowband and broadband channel measurements are introduced. Section 3 shows the fitted path loss models in narrowband measurements. In Section 4, the broadband channel parameters including PDP, RMS-DS, RMS-AS, eigenvalue, and capacity are analyzed, whereas Section 5 draws conclusions.

\section{Channel Measurements}

2.1. Measurement Environment Description. As depicted in Figure 1(a), the measurements were conducted in a metro Line 7 between Shanghai University and QiHua Rd. in Shanghai, China. The cross section of the tunnel is arched, with a radius of $2.78 \mathrm{~m}$, a height of $5 \mathrm{~m}$, and a width of $3.4 \mathrm{~m}$ at the bottom, as given in Figure 1(b). The tunnel wall materials are reinforced with concrete material. As shown in Figure 1(c), the tunnel can be divided into three sections structurally, S1 is a short straight tunnel with a length of $20 \mathrm{~m}, \mathrm{~S} 2$ is a curve with a length of $100 \mathrm{~m}$ and a radius of curvature of $1500 \mathrm{~m}$, and S3 is a long straight tunnel with a length of $700 \mathrm{~m}$. In our narrowband measurements, the farthest measurement distance between $\mathrm{Tx}$ and $\mathrm{Rx}$ is $800 \mathrm{~m}$. In wideband measurements, due to the busy schedule of the subway line, we only measured the first $500 \mathrm{~m}$ broadband channels. Within this $500 \mathrm{~m}$ measurement range, 91 measurement points are sampled at $3.5 \mathrm{GHz}$, while 43 measurement points are sampled at $5.6 \mathrm{GHz}$.

As shown in Figure 2, to simulate the actual base station, the $\mathrm{Tx}$ is fixed at the end of the extension section of the platform, close to the tunnel wall. The Tx is $1.8 \mathrm{~m}$ high, and the platform height is calculated as $1.3 \mathrm{~m}$, where the $\mathrm{Rx}$ is located on a mobile trolley on the central rail of the tunnel, simulating as an access point. In order to reduce the interference of testers on signal transmission, we increased the height of the $\mathrm{Rx}$ to $2.7 \mathrm{~m}$.

2.2. Narrowband Measurement System. In the narrowband measurements, as shown in Figure 3, the signal generator Agilent E8257D and spectrum analyzer Ceyear 4024G are separately employed in both sides of Tx and $\mathrm{Rx}$, respectively. Ultra-wideband $(1 \mathrm{GHz}-40 \mathrm{GHz})$ omnidirectional antennas are equipped on both sides, and high-precision rubidium clocks are used to ensure synchronization. An uninterruptible power supply (UPS) ensures the power supply of the equipment in the whole measurement process. Five potential $5 \mathrm{G}$ frequency bands are investigated in our narrowband measurements, including $2.6 \mathrm{GHz}, 3.5 \mathrm{GHz}$, $5.6 \mathrm{GHz}, 10 \mathrm{GHz}$, and $28 \mathrm{GHz}$.

2.3. Broadband Measurement System. In the broadband measurements, the pseudo-noise (PN) sequence correlation method is used. At the Tx side, a PN sequence is modulated with a bandwidth of $160 \mathrm{MHz}$ and a length of 1023 chips on the carrier frequencies of $3.5 \mathrm{GHz}$ and $5.6 \mathrm{GHz}$ by Binary Phase Shift Keying (BPSK). The Rx is divided into 8 channels to collect data in parallel. We use a low-noise amplifier to improve the received signal-to-noise ratio (SNR). Note that it takes only $10 \mathrm{~ms}$ to complete the static channel measurements of $2048(32 \times 64)$ subchannels in one cycle, and 

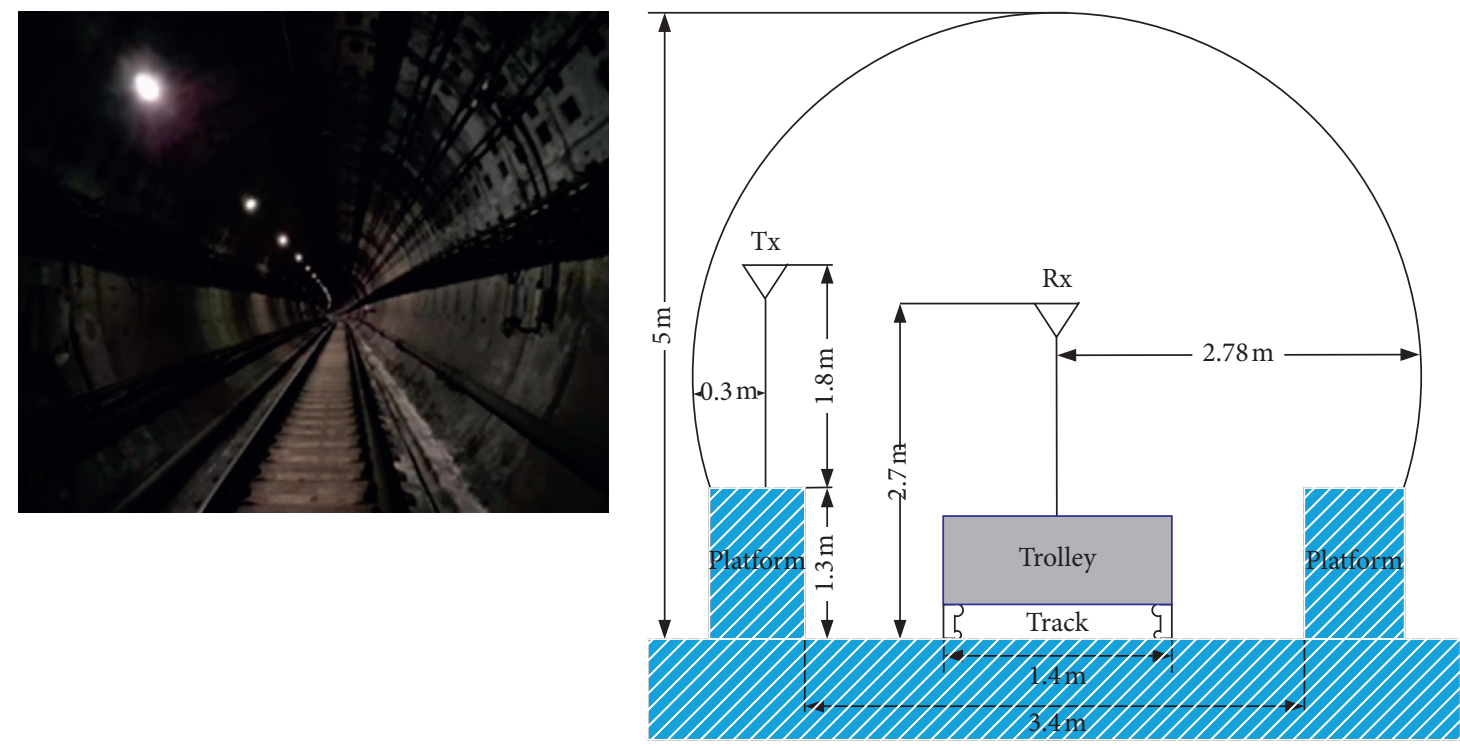

(a)

(b)

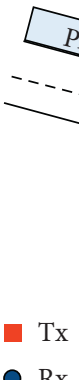

(c)

FIgURE 1: Measurement environment. (a) Real scene. (b) Cross section. (c) Floor map.

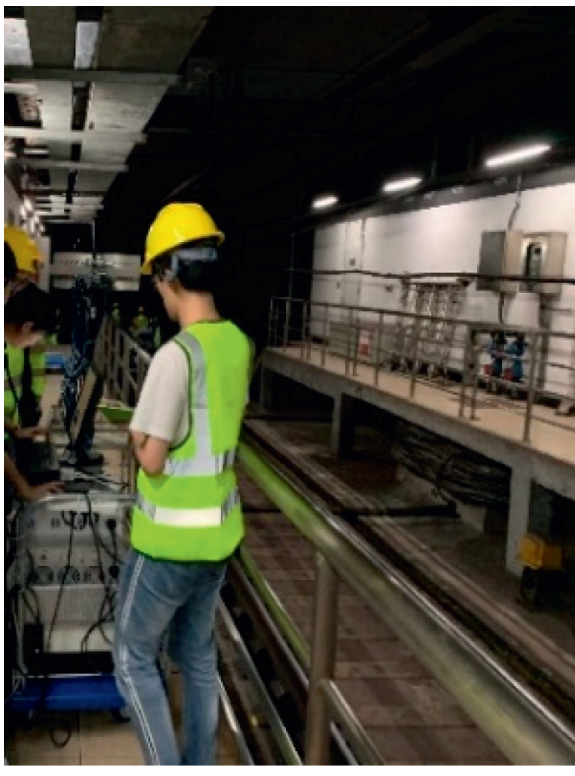

(a)

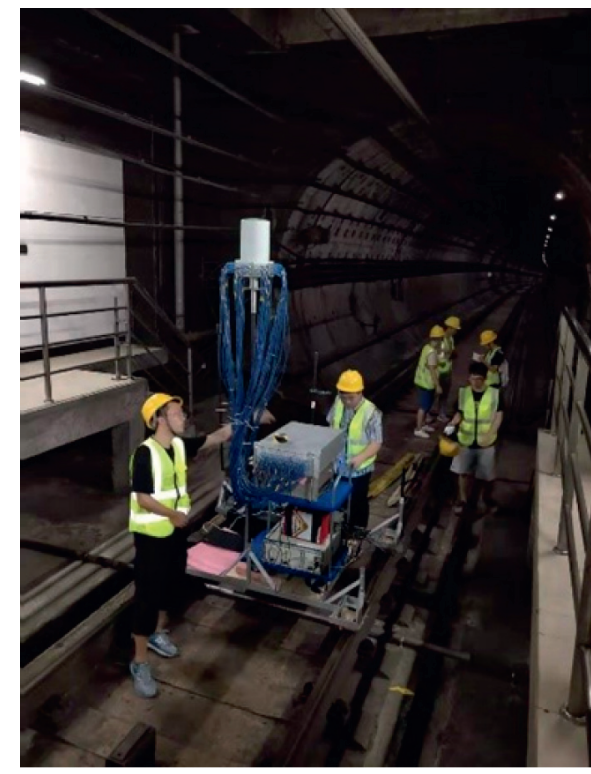

(b)

FIgURE 2: Real measurement scenario. (a) Tx side. (b) Rx side. 


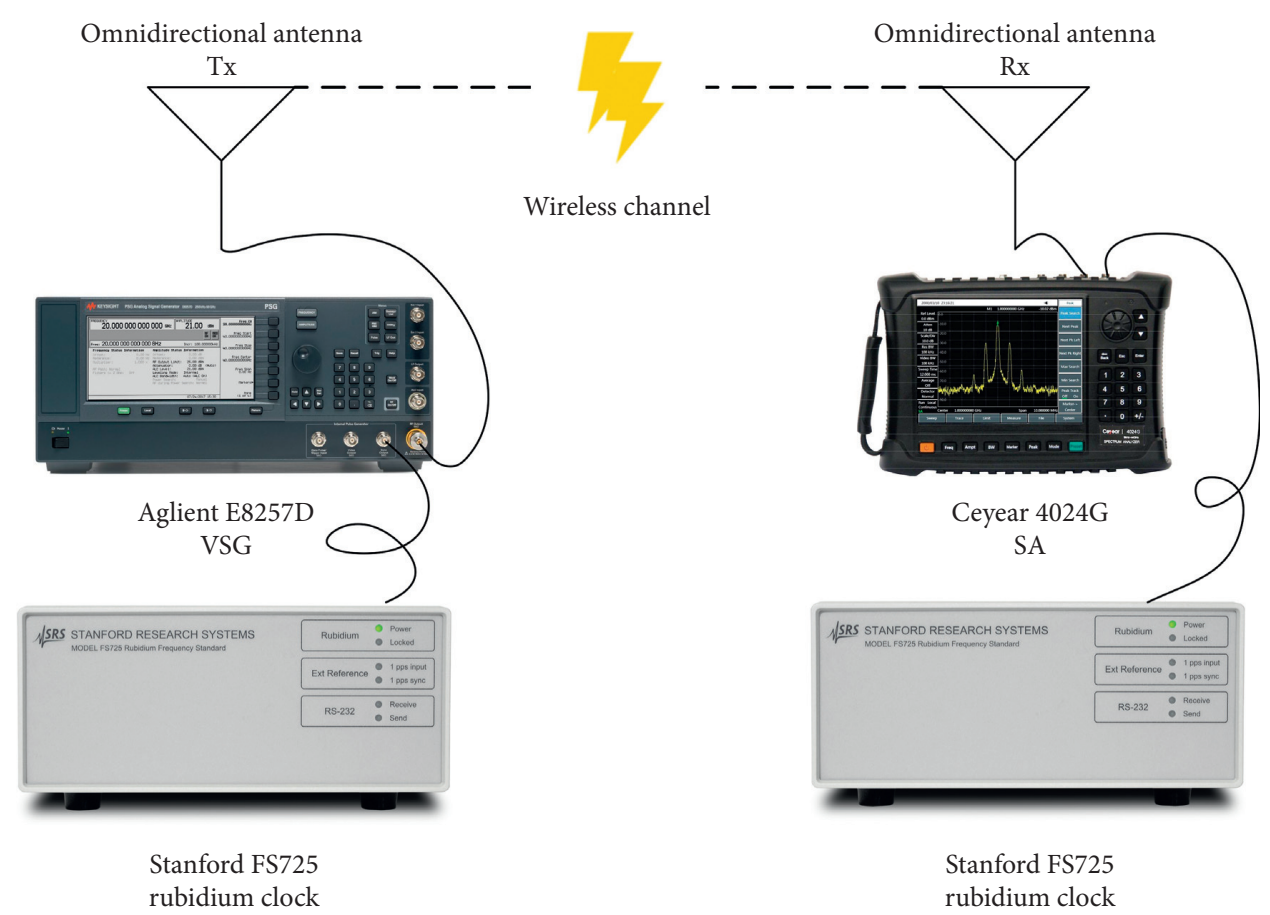

FIGURE 3: Narrowband measurements setup description.

128 cycles of data are collected at each $\mathrm{Rx}$ measurement position.

In the broadband measurements, directional patch antenna arrays are equipped at both $\mathrm{Tx}$ and $\mathrm{Rx}$ sides. The $3 \mathrm{~dB}$ beamwidth of elements on the vertical and horizontal planes is $100^{\circ}$ and $120^{\circ}$, respectively. All multipath signal propagation paths in the tunnel environment can be detected perfectly. The structure of the $\mathrm{Tx}$ and $\mathrm{Rx}$ antenna is very similar to that used in $[24,25]$, as shown in Figure 4 . The Tx is equipped with a $2 \times 8$ uniform rectangular antenna patch array. On the Rx side, the uniform cylindrical array can be divided into 8 sectors, and each sector has 4 antenna patches arranged in the vertical direction. The antenna patch spacing is half of the wavelength, and each antenna pair includes a pair of colocated dual-polarized antennas. Each patch antenna encompasses two elements with the main polarization of $\pm 45^{\circ}$ at $3.5 \mathrm{GHz}$ and vertical and horizontal at $5.6 \mathrm{GHz}$. The important measurement parameters are summarized in Table 1 .

\section{Narrowband Channel Characterization}

3.1. Path Loss. Path loss characterizes the signal energy attenuation after the propagation of transmitted signals. Figure 5 presents the path loss measured in the given subway tunnel environment. The most typical path loss model is first used to quantitatively analyze the path loss parameters [26]:

$$
P L[d B]=P L\left(d_{0}\right)+10 n \log _{10}\left(\frac{d}{d_{0}}\right)+X_{\sigma},
$$

where $d_{0}$ is a reference distance and usually set to $1 \mathrm{~m}$ in a tunnel scene, $d$ is the distance between the Tx and $\mathrm{Rx}$, and $n$ is the path loss index and equals to 2 in free space. $X_{\sigma}$ is a random variable with zero mean Gaussian distribution and the standard deviation is $\sigma . P L\left(d_{0}\right)$ is optimized with the path loss index $n$ to minimize the mean-square error (MSE) between model and the real measurements. The extracted parameters are listed in Table 2. It can be found that the path loss exponent at all measured frequency bands in the tunnel is smaller than that of a free space channel and decreases with the increment of signal frequency. This is because the waveguide effect in the tunnel still exists on the whole. Furthermore, the shorter the wavelength of the signal, the greater the possibility of specular reflection on the tunnel, which enhances the waveguide effect of the tunnel on higher frequency signals.

Meanwhile, it is worth noting that the line-of-sight (LOS) propagation can be blocked by the curved tunnel wall (S2); thus, the location where the LOS components are blocked is very important to accurately predict the path loss over there [27]. Based on the geometric representation of the location for break point as shown in Figure 6, we estimate that the break point $d_{b p}$ of LOS and non-line-of-sight (NLOS) propagation of the tunnel in this article is about $91 \mathrm{~m}$. The two-slope path loss model under the tunnel can be defined as

$$
P L[d B]= \begin{cases}P L\left(d_{0}\right)+10 n_{1} \log _{10}\left(\frac{d}{d_{0}}\right)+X_{\sigma 1}, & d \leq d_{b p}, \\ P L\left(d_{b p}\right)+10 n_{2} \log _{10}\left(\frac{d}{d_{b p}}\right)+X_{\sigma 2}, & d>d_{b p} .\end{cases}
$$

Figure 7 shows the two-slope path loss model at $28 \mathrm{GHz}$. The coefficients of the models for all frequency bands are 


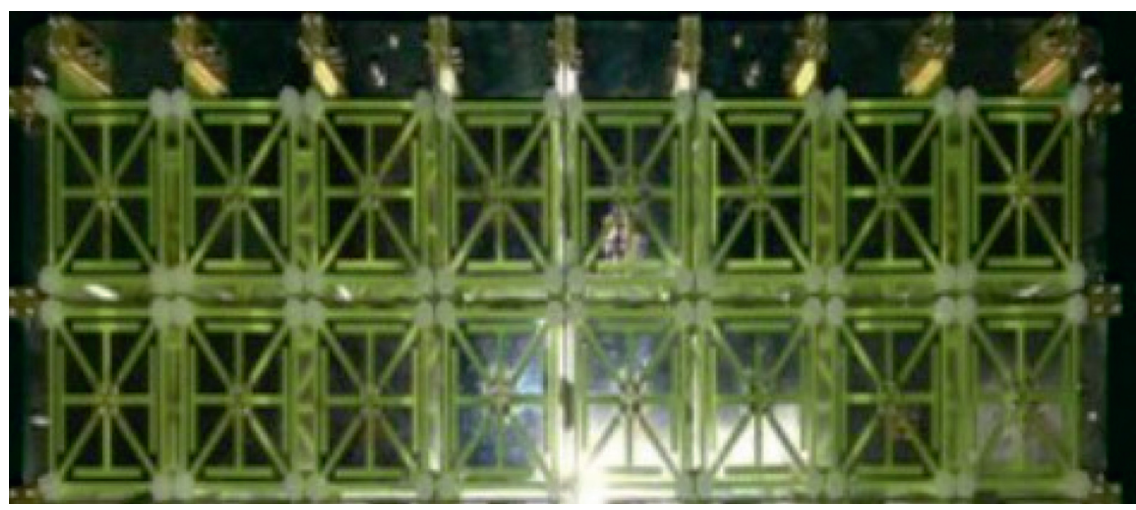

(a)

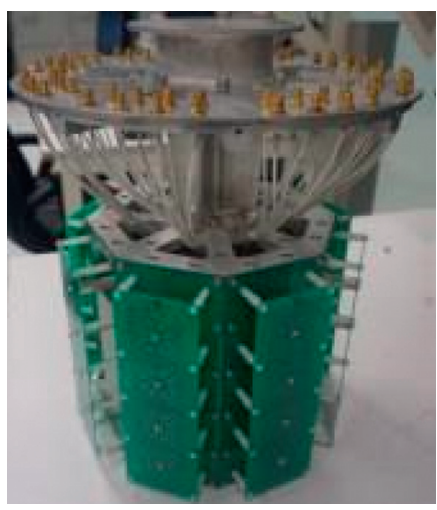

(b)

Figure 4: Antenna array used in the measurements. (a) Tx. (b) Rx.

TABLE 1: Measurement parameters.

\begin{tabular}{|c|c|c|}
\hline Parameters & Narrowband measurement & Broadband measurement \\
\hline Frequency & $2.6 \mathrm{GHz} / 3.5 \mathrm{GHz} / 5.6 \mathrm{GHz} / 10 \mathrm{GHz} / 28 \mathrm{GHz}$ & $3.5 \mathrm{GHz} / 5.6 \mathrm{GHz}$ \\
\hline Probe signal & Sine wave & PN sequence \\
\hline Bandwidth & 0 & $160 \mathrm{MHz}$ \\
\hline Antenna structure & $\begin{array}{cc}\text { Omnidirectional antenna } & \text { Omnidirectional antenna } \\
(\mathrm{Tx}) & (\mathrm{Rx})\end{array}$ & $\begin{array}{cc}\text { Uniform rectangular } & \text { Uniform cylindrical } \\
\text { array }(\mathrm{Tx}) & \text { array }(\mathrm{Rx})\end{array}$ \\
\hline Polarization of $\mathrm{Tx} / \mathrm{Rx}$ antenna & Vertical/vertical & $\begin{array}{c}\text { Dual polarization: } 3.5 \mathrm{GHz}: \pm 45^{\circ} 5.6 \mathrm{GHz} \text { : } \\
\text { vertical and horizontal }\end{array}$ \\
\hline Height of $\mathrm{Tx} / \mathrm{Rx}$ antenna & $1.8 \mathrm{~m} / 2.7 \mathrm{~m}$ & $1.8 \mathrm{~m} / 2.7 \mathrm{~m}$ \\
\hline Farthest measurement distance & $800 \mathrm{~m}$ & $500 \mathrm{~m}$ \\
\hline Number of sampling points & 85 & 3.5 GHz: $915.6 \mathrm{GHz}: 43$ \\
\hline
\end{tabular}

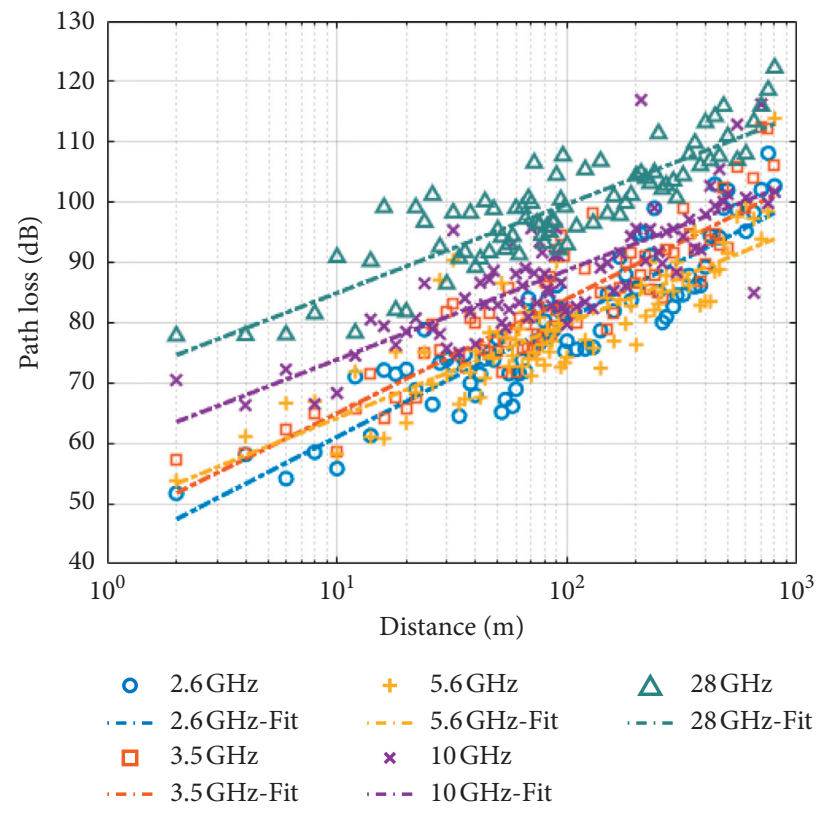

Figure 5: Path loss at different frequencies. 
TABle 2: Path loss coefficients.

\begin{tabular}{lccr}
\hline Frequency $(\mathrm{GHz})$ & $P L\left(d_{0}\right)(\mathrm{dB})$ & $n$ & $\sigma(\mathrm{dB})$ \\
\hline 2.6 & 41.55 & 1.95 & 5.18 \\
3.5 & 46.01 & 1.90 & 5.06 \\
5.6 & 48.59 & 1.56 & 5.63 \\
10 & 59.07 & 1.48 & 5.85 \\
28 & 70.19 & 1.47 & 4.53 \\
\hline
\end{tabular}

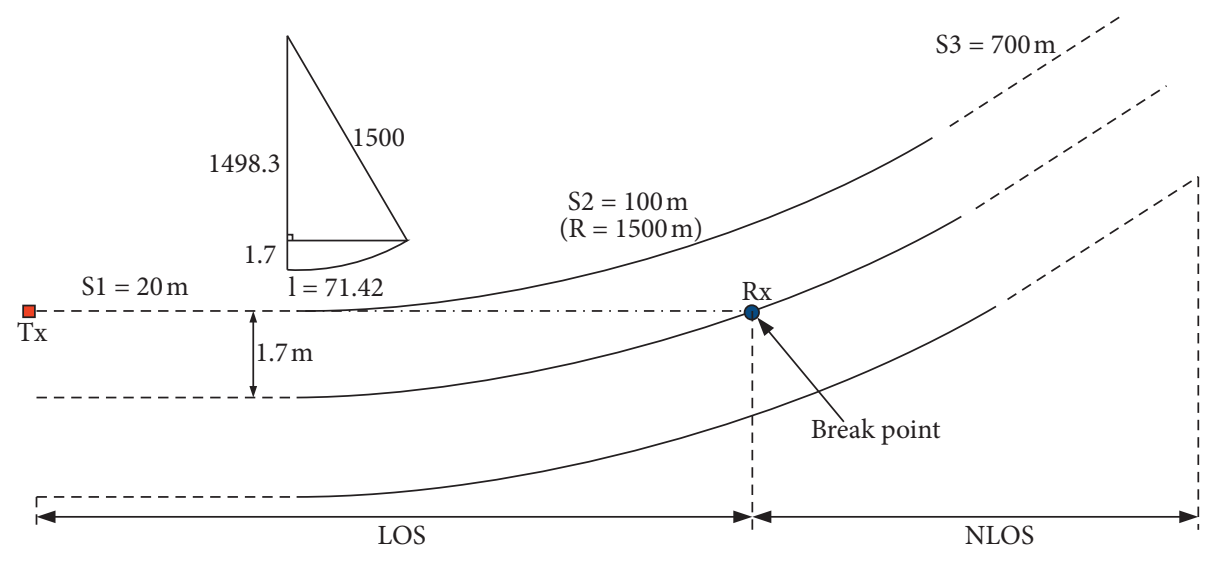

Figure 6: The location for break point.

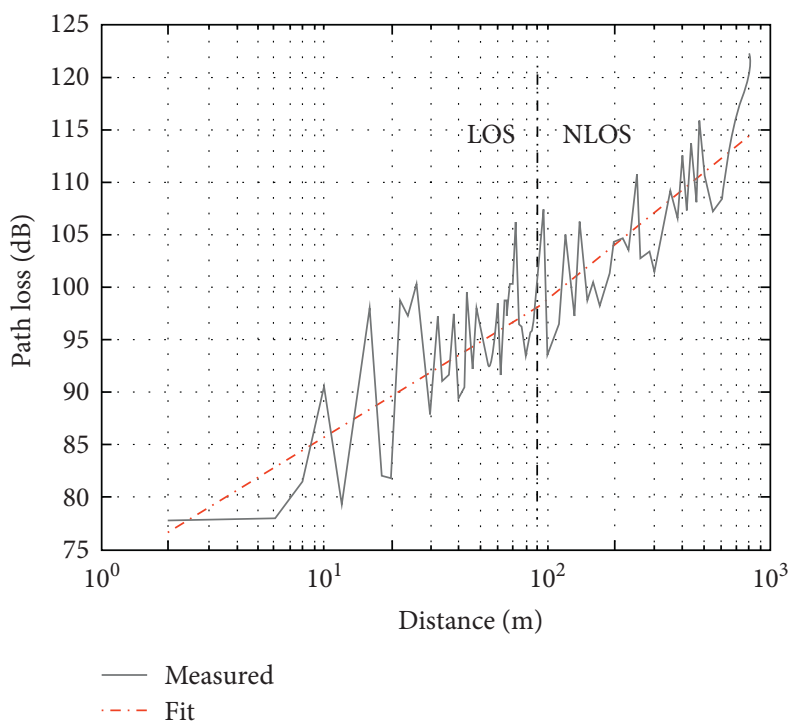

Figure 7: Measured data versus two-slope path loss model at $28 \mathrm{GHz}$.

listed in Table 3. The path loss exponent varies from 1.06 to 1.80 in the LOS propagation scenario, while in the NLOS scenario, the path loss exponent ranges from 1.67 to 2.42 . This shows that before the break point, the multipath propagates in the axial direction of the tunnel and propagates in the way of reflection between the tunnel walls, resulting in a smaller propagation loss. When the curved tunnel wall occludes the multipath components, the above waveguide effect is weakened. Compared with the path loss models in [27], it shows a smaller path loss exponent in the NLOS area mainly because the radius of curvature of $1500 \mathrm{~m}$ is much larger than that in [27]. In addition, Tx is placed close to the tunnel wall in our measurements, while Tx and $\mathrm{Rx}$ in [27] are set in the center of a tunnel. From the comparison of 5 measured signal frequencies, the propagation attenuation rate of $5.6 \mathrm{GHz}$ is the smallest in the LOS scenario while the path loss exponents of $10 \mathrm{GHz}$ and $28 \mathrm{GHz}$ are smaller in the NLOS scenario. 
TABLE 3: Path loss coefficients of two-slope models.

\begin{tabular}{|c|c|c|c|c|c|c|}
\hline \multirow{2}{*}{ Frequency $(\mathrm{GHz})$} & \multicolumn{3}{|c|}{ LOS } & \multicolumn{3}{|c|}{ NLOS } \\
\hline & $P L\left(d_{0}\right)(\mathrm{dB})$ & $n_{1}$ & $\sigma_{1}(\mathrm{~dB})$ & $P L\left(d_{b p}\right)(\mathrm{dB})$ & $n_{2}$ & $\sigma_{2}(\mathrm{~dB})$ \\
\hline 2.6 & 47.17 & 1.57 & 4.75 & 77.85 & 2.42 & 5.27 \\
\hline 3.5 & 47.29 & 1.80 & 4.20 & 82.46 & 2.09 & 5.87 \\
\hline 5.6 & 56.20 & 1.06 & 5.89 & 76.92 & 2.09 & 4.82 \\
\hline 10 & 59.90 & 1.41 & 4.75 & 87.45 & 1.67 & 6.77 \\
\hline 28 & 72.90 & 1.29 & 4.83 & 98.11 & 1.72 & 4.06 \\
\hline
\end{tabular}

\section{Broadband Channel Characterization}

4.1. PDP and RMS-DS. PDP is frequently used to characterize the distribution characteristics of multipaths in the delay domain. Average Power Delay Spectrum (APDP) is the average of PDPs over all Tx-Rx links, which can reduce the interference of small-scale fading on effective signal multipaths [28], expressed as

$$
\operatorname{APDP}(\tau)=\frac{1}{N_{T} N_{R}} \sum_{m=1}^{N_{T}} \sum_{n=1}^{N_{R}}\left|h_{m n}(\tau)\right|^{2},
$$

where $N_{T}$ and $N_{R}$ represent the number of array elements of Tx and $\mathrm{Rx}$, respectively. $h_{m n}(\tau)$ is the complex channel impulse response between the $m$-th Tx and the $n$-th Rx.

Figure 8 shows normalized APDP at all measurement points in the tunnel scenario. Taking APDP at $3.5 \mathrm{GHz}$ as an example, the multipath component decays very quickly with time. If $-20 \mathrm{~dB}$ is chosen as the power threshold of the multipath components, the delay of the multipath component will rapidly attenuate from $100 \mathrm{~ns}$ to $50 \mathrm{~ns}$ with the increase of Tx-Rx distance. The trend of APDP at $5.6 \mathrm{GHz}$ is observed roughly the same as that of $3.5 \mathrm{GHz}$.

We can further study the delay spread by APDP. Delay spread is a statistical description of the delay characteristics of a multipath channel, which determines the highest transmission rate supported by the channel, in case of no intersymbol interference within a broadband communication system. RMS-DS is usually defined as the central second moment of APDP, expressed as follows [26]:

$$
\tau_{r m s}=\sqrt{\frac{\sum_{k=1}^{K} P\left(\tau_{k}\right) \cdot\left(\tau_{k}\right)^{2}}{\sum_{k=1}^{K} P\left(\tau_{k}\right)}-\left(\frac{\sum_{k=1}^{K} P\left(\tau_{k}\right) \cdot\left(\tau_{k}\right)}{\sum_{k=1}^{K} P\left(\tau_{k}\right)}\right)^{2}},
$$

where $K$ represents the number of effective multipath components. $P\left(\tau_{k}\right)$ and $\tau_{k}$ are the estimated power and the relative delay of the $k$-th effective multipath.

It is observed from Figure 9(a) that RMS-DS decreases with the increase of $\mathrm{Tx}-\mathrm{Rx}$ distance, which is basically consistent with the simulation results of the ray-tracing method in [15]. This can be explained by the modal theory [29]. When Tx-Rx distance is short, multiple propagation modes are excited. As the distance increases, especially after entering the NLOS propagation scenario, the high-order modal attenuation is severe, and only the low-order basic modal is maintained. Therefore, the RMS-DS in the NLOS region is relatively stable and changes smoothly. The statistic and percentiles are listed in Table 4. Except for the LOS scenario when the Tx-Rx distance is short, RMS-DS varies from $6 \mathrm{~ns}$ to $10 \mathrm{~ns}$ at $3.5 \mathrm{GHz}$, while RMS-DS at $5.6 \mathrm{GHz}$ changes from $4 \mathrm{~ns}$ to $8 \mathrm{~ns}$. Overall, it can be concluded from Figure 9(b) that the RMS-DS at $3.5 \mathrm{GHz}$ is slightly larger than that of $5.6 \mathrm{GHz}$.

4.2. RMS-AS. RMS-AS is usually explored to indicate the expansion of the departure or arrival angle of signals on the antenna due to multipath reflection and scattering effects. In this paper, we use the Bartlett beamforming method to estimate the angle characteristics in azimuth of arrival (AOA), the elevation of arrival (EOA), azimuth of departure (AOD), and elevation of departure (EOD). RMS-AS is defined as the second-order central moment of the angle spectrum and can be calculated as follows [26]:

$$
\begin{aligned}
\Delta_{r m s} & =\sqrt{\frac{\sum_{k=1}^{K}\left(\theta_{k}-\bar{\theta}\right)^{2} P\left(\theta_{k}\right)}{\sum_{k=1}^{K} P\left(\theta_{k}\right)},} \\
\bar{\theta} & =\frac{\sum_{k=1}^{K} \theta_{k} P\left(\theta_{k}\right)}{\sum_{k=1}^{K} P\left(\theta_{k}\right)},
\end{aligned}
$$

where $\theta_{k}$ is the angle of the $k$-th effective multipath.

Figure 10 shows the angle-delay power spectrum at a typical LOS position (Tx-Rx distance of $15 \mathrm{~m}$ ) and NLOS position ( $\mathrm{Tx}-\mathrm{Rx}$ distance of $400 \mathrm{~m}$ ) in AOA. It can be observed that at a typical LOS measurement location, most of the multipaths are concentrated in the LOS (about $-90^{\circ}$ ) direction, and the other multipaths can be received by $\mathrm{Rx}$ in the directions of $-60^{\circ}$ and $-100^{\circ}$, reflected from the walls of both sides of the tunnel. At the NLOS location, due to the limited propagation space of the tunnel, the waveguide effect forces the multipath components to propagate along the direction of the track. The angles for approaching the receiver are basically the same, all around $-80^{\circ}$.

It is found that the RMS-AS decreases rapidly with the increase of the Tx-Rx distance from Figure 11. The statistical results of all RMS-AS are summarized in Table 5. The uniform cylindrical array at the $\mathrm{Rx}$ side can receive signals from different azimuth angles of each sector, and a larger expansion of the angle of arrival can be obtained. About $90 \%$ of the AOD and EOD is less than $10^{\circ}$ at the Tx side, and $90 \%$ of EOA is less than $5^{\circ}$. Comparing the RMS-AS at different frequency bands, it can be concluded that the RMS-AS at $3.5 \mathrm{GHz}$ is larger than that of $5.6 \mathrm{GHz}$ except in AOA. The 


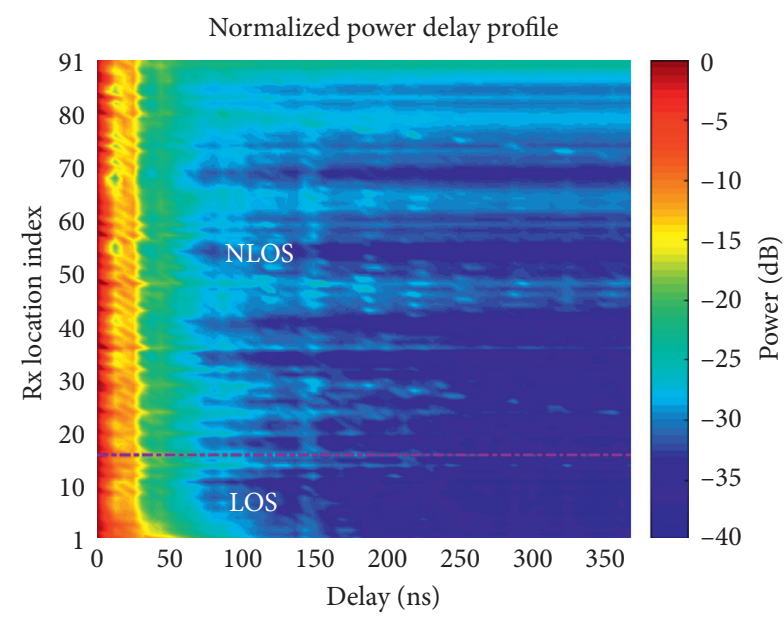

(a)

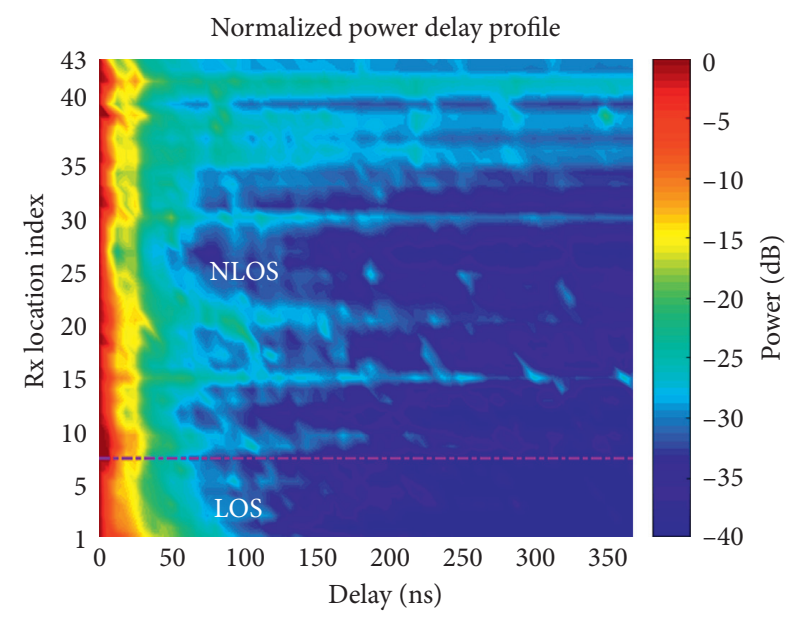

(b)

Figure 8: Normalized APDP. (a) $3.5 \mathrm{GHz}$. (b) $5.6 \mathrm{GHz}$.

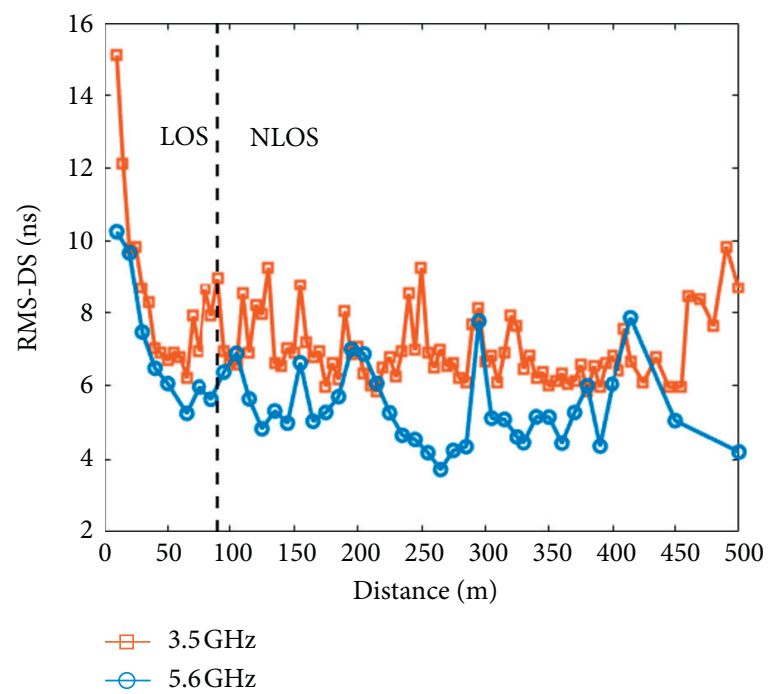

(a)

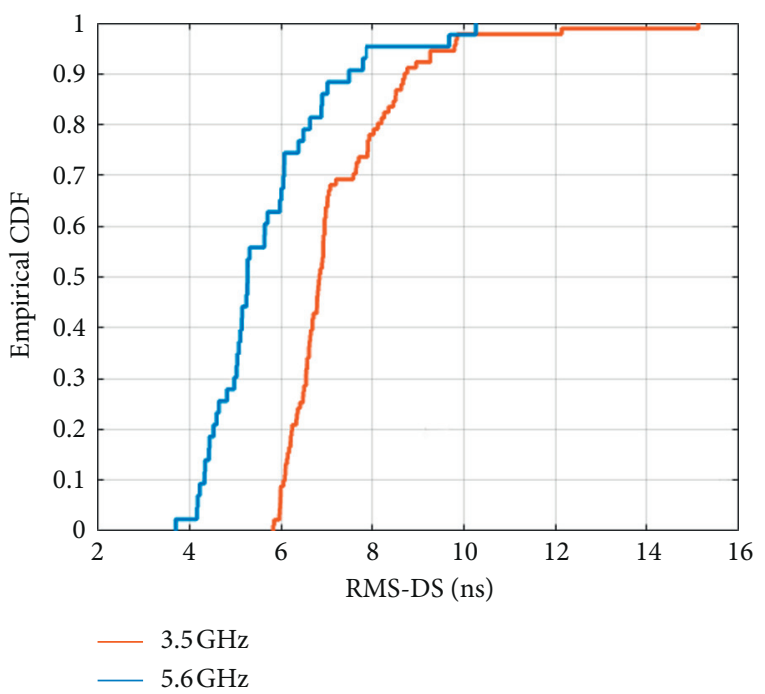

(b)

Figure 9: RMS-DS. (a) Variation with Tx-Rx distance. (b) CDF.

TABLE 4: Statistic of RMS-DS.

\begin{tabular}{lccccc}
\hline Frequency $(\mathrm{GHz})$ & $10 \%(\mathrm{~ns})$ & $50 \%(\mathrm{~ns})$ & $90 \%(\mathrm{~ns})$ & Mean $(\mathrm{ns})$ & Standard deviation $(\mathrm{ns})$ \\
\hline 3.5 & 6.09 & 6.84 & 8.72 & 7.26 & 1.40 \\
5.6 & 4.34 & 5.28 & 7.48 & 5.70 & 1.40 \\
\hline
\end{tabular}

above experimental conclusions are quite beneficial to the deployment and design of antennas in tunnel scenarios.

4.3. Eigenvalue and Channel Capacity. We estimate the performance of the wireless communication system by measuring the channel capacity and the eigenvalue distribution of the channel matrix. The complex channel matrix $\mathrm{H}$ can be expressed as follows:

$$
H=\left(\begin{array}{ccc}
\widetilde{h_{11}} & \cdots & \widetilde{h_{1 N_{R}}} \\
\vdots & \ddots & \vdots \\
\widetilde{h_{N_{T}} 1} & \cdots & \widetilde{h_{N_{T} N_{R}}}
\end{array}\right),
$$




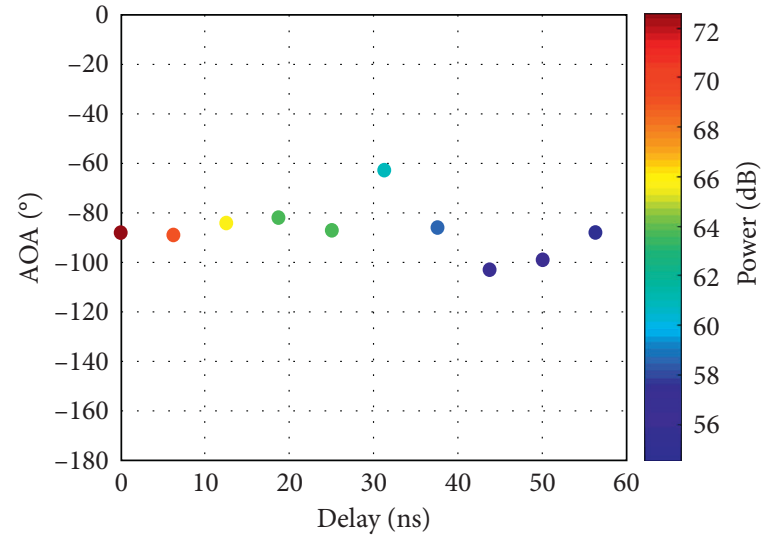

(a)

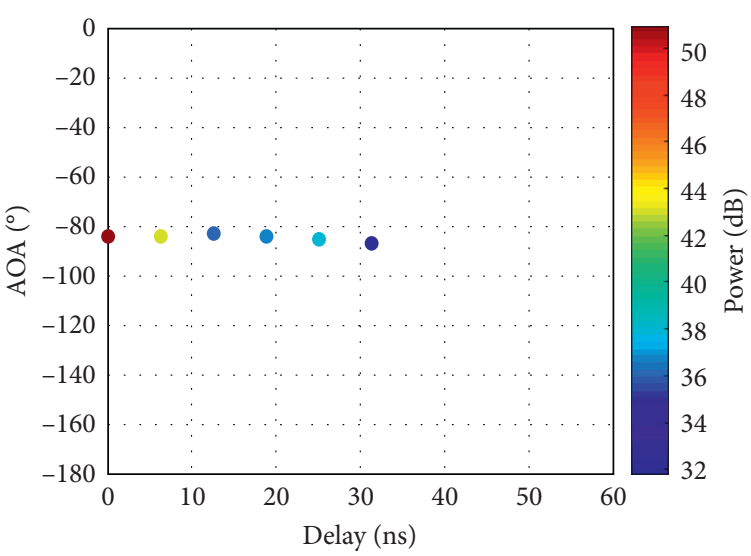

(b)

Figure 10: Multipath component angle-delay power spectrum at 3.5 GHz in AOA. (a) At a typical LOS position (Tx-Rx distance of $15 \mathrm{~m}$ ). (b) At a typical NLOS position (Tx-Rx distance of $400 \mathrm{~m}$ ).

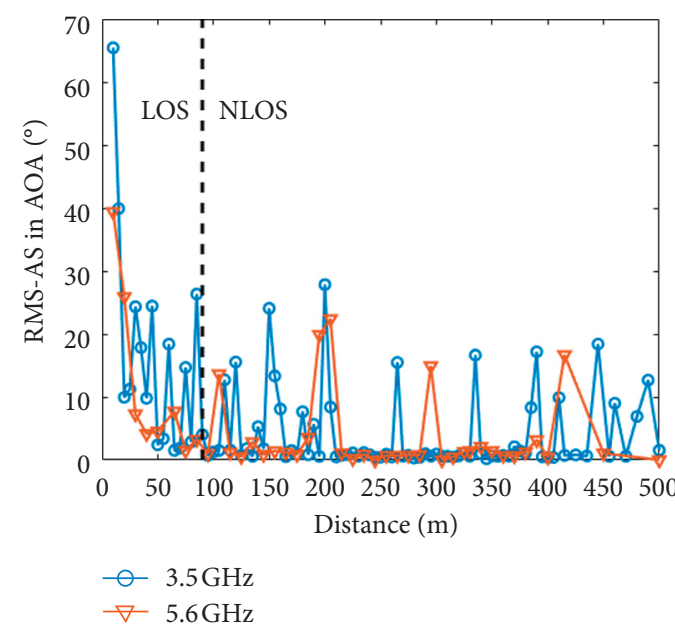

(a)

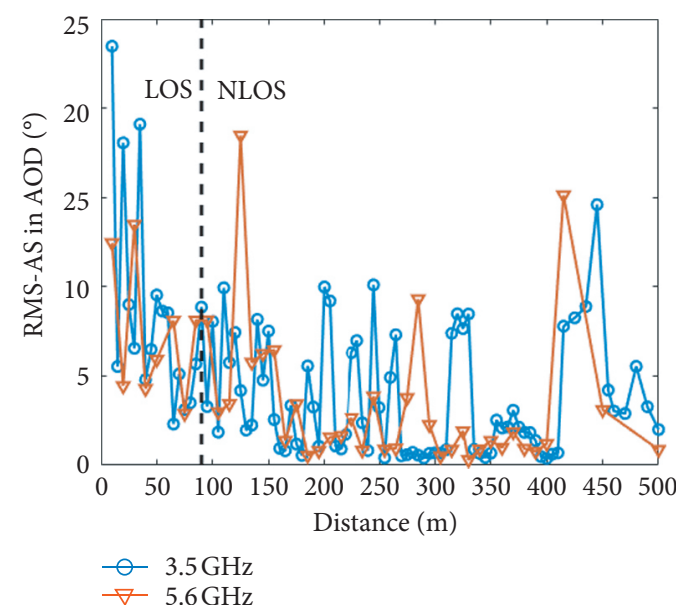

(b)

Figure 11: RMS-AS variation with Tx-Rx distance. (a) AOA. (b) AOD.

TABLE 5: Statistics of RMS-AS.

\begin{tabular}{lcccccc}
\hline Frequency $(\mathrm{GHz})$ & Angle & $10 \%\left(^{\circ}\right)$ & $50 \%\left(^{\circ}\right)$ & $90 \%\left(^{\circ}\right)$ & Mean $\left(^{\circ}\right)$ & Standard deviation $\left(^{\circ}\right)$ \\
\hline \multirow{4}{*}{3.5} & AOA & 0.47 & 1.16 & 17.83 & 6.15 & 9.74 \\
& EOA & 0.49 & 1.20 & 3.78 & 2.01 & 2.61 \\
& AOD & 0.56 & 3.08 & 9.00 & 4.48 & 4.35 \\
5.6 & EOD & 1.83 & 5.34 & 9.42 & 5.52 & 2.90 \\
& AOA & 0.22 & 1.25 & 16.68 & 4.96 & 8.34 \\
& EOA & 0.71 & 1.66 & 4.63 & 2.36 & 4.92 \\
& AOD & 0.77 & 2.65 & 9.30 & 4.09 & 4.30 \\
\hline
\end{tabular}

where $\widetilde{h_{m n}}$ can be obtained by summing the multipath signals of each subchannel:

$$
\widetilde{h_{m n}}=\sum_{k=1}^{K} h_{m n}\left(\tau_{k}\right) \text {. }
$$

The normalized eigenvalue $\lambda i$ of $\mathrm{HH}^{\dagger}$ is seen in Figure 12. Here, $(\cdot)^{\dagger}$ is the conjugate transpose of the matrix. It can be found that the attenuation of the channel matrix eigenvalues at a typical LOS position (Tx-Rx distance of $15 \mathrm{~m}$ ) is slower than that of NLOS position (Tx-Rx distance of $400 \mathrm{~m}$ ). The 


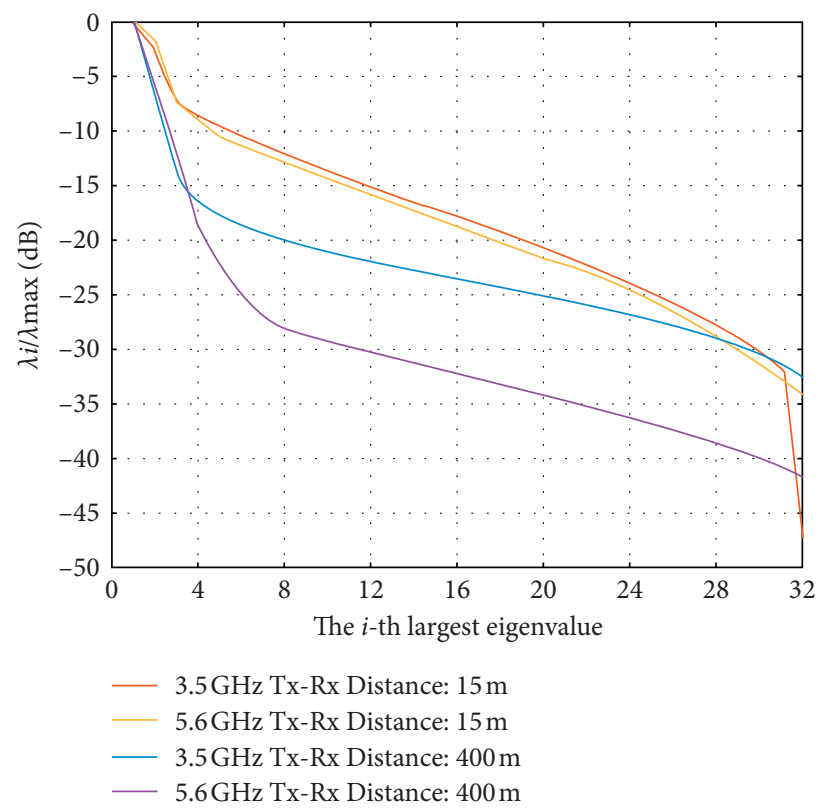

Figure 12: Normalized eigenvalues at a typical LOS scenario and NLOS scenario.

normalized eigenvalue of $5.6 \mathrm{GHz}$ has greater attenuation than that of $3.5 \mathrm{GHz}$. Assuming that $-16 \mathrm{~dB}(0.025)$ is used as the eigenvalue threshold, it is considered that eigenvalues that are smaller than the threshold value have little effect on the channel matrix. Taking the condition of $\mathrm{Tx}-\mathrm{Rx}$ distance of $15 \mathrm{~m}$ at $3.5 \mathrm{GHz}$ as an example, the number of effective eigenvalues of the channel matrix is only 12 , which is much smaller than the minimum number of $\mathrm{Tx}$ and $\mathrm{Rx}$ antenna elements $\min \left(N_{T}, N_{R}\right)$. It shows that in the measured tunnel environment, the massive MIMO channel matrix has a serious rank reduction, which will have an adverse effect on the multistream parallel transmissions in wireless communication systems.

Channel capacity refers to the maximum information rate transmitted in an error-free channel, which is one of the most important indicators for measuring wireless propagation channels. It can be expressed as [30]

$$
C=\log _{2} \operatorname{det}\left[I_{N_{R}}+\frac{\rho}{N_{T}} H_{n} H_{n}^{\dagger}\right]
$$

where $I_{N_{R}}$ is the real identity matrix. $\rho$ is the SNR at the Rx side, which is set to $10 \mathrm{~dB}$ here. $H_{n}$ is the normalized channel matrix from which the channel complex matrix $\mathrm{H}$ removes the influence of path loss and satisfies $\left\|H_{n}\right\|_{F}^{2}=N_{T} N_{R}$.

In order to explore the deployment prospects of massive MIMO in subway tunnel scenarios, we focus on the influence of the Tx-Rx distance and the number of transmitting antenna elements on the channel capacity. Under the condition of a constant $S N R=10 \mathrm{~dB}$ and a fixed number $N_{R}=64$ of cylindrical antenna elements at the $\mathrm{Rx}$ side, channel capacity at $3.5 \mathrm{GHz}$ and $5.6 \mathrm{GHz}$ is shown in Figures 13(a) and 13(b), respectively.

Figure $13(\mathrm{c})$ shows the $32 \times 64$ MIMO channel capacity with the Tx-Rx distance in the tunnel scenario at $3.5 \mathrm{GHz}$ and $5.6 \mathrm{GHz}$. It can be seen that, under the propagation condition of LOS, the channel capacity decreases rapidly with the increase of the $\mathrm{Tx}-\mathrm{Rx}$ distance. Consider the $3.5 \mathrm{GHz}$ channel capacity as an example; it drops from $85 \mathrm{bits} / \mathrm{s} / \mathrm{Hz}$ to $45 \mathrm{bits} / \mathrm{s} / \mathrm{Hz}$. The average channel capacities of $3.5 \mathrm{GHz}$ and $5.6 \mathrm{GHz}$ are $54.96 \mathrm{bits} / \mathrm{s} / \mathrm{Hz}$ and $55.55 \mathrm{bits} / \mathrm{s} /$ $\mathrm{Hz}$ in the LOS propagation scenario, respectively. Channel capacity at $5.6 \mathrm{GHz}$ appears to be slightly higher than that at $3.5 \mathrm{GHz}$ in the LOS scenario. This can be explained as the higher the frequency is, the more modalities are excited, which enhances the decorrelation at the receiver side. When the $\mathrm{Rx}$ enters the NLOS area, the number of active modes decays rapidly and the channel capacity attenuates slowly. The average channel capacity of $3.5 \mathrm{GHz}$ and $5.6 \mathrm{GHz}$ in the NLOS area is $36.24 \mathrm{bits} / \mathrm{s} / \mathrm{Hz}$ and $28.96 \mathrm{bits} / \mathrm{s} / \mathrm{Hz}$, respectively. From the ray theory point of view, in the LOS area, the direct component exists stably. As the distance between $\mathrm{Tx}$ and $\mathrm{Rx}$ increases, the distance between the multipath component reflected by the tunnel wall and the direct component gradually decreases, resulting in smaller RMSDS and RMS-AS. The similarity between multipath components is increased, thereby reducing the ability of channel diversity gain. In the NLOS propagation area, the direct component is blocked by the tunnel curvature, and the surface of the curved tunnel wall will provide irregular reflection. The propagation distance between multipath components is relatively stable, so the channel capacity does not change significantly with the distance between Tx and 


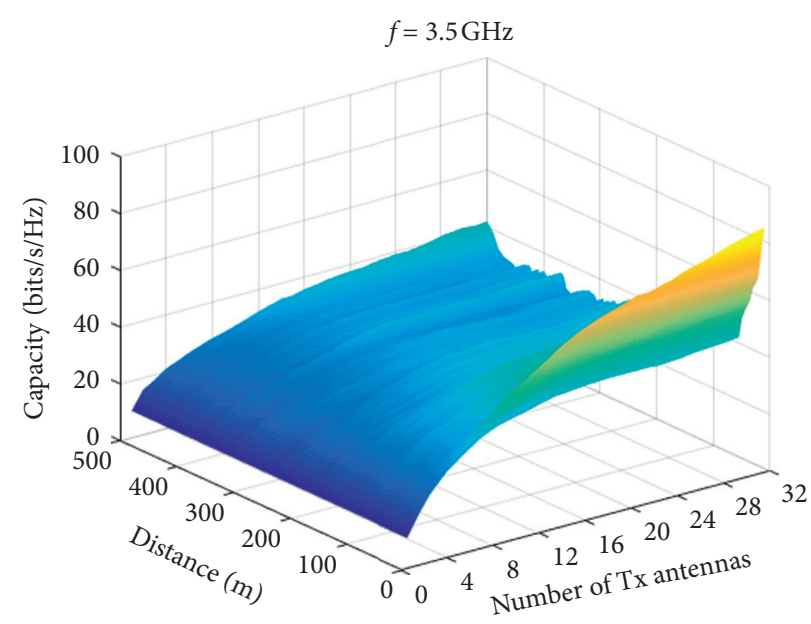

(a)

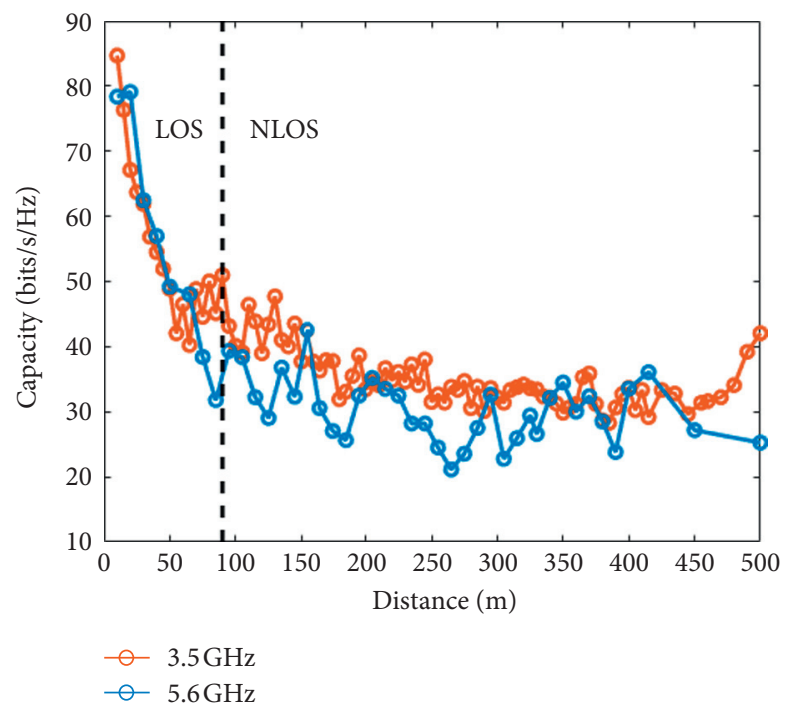

(c)

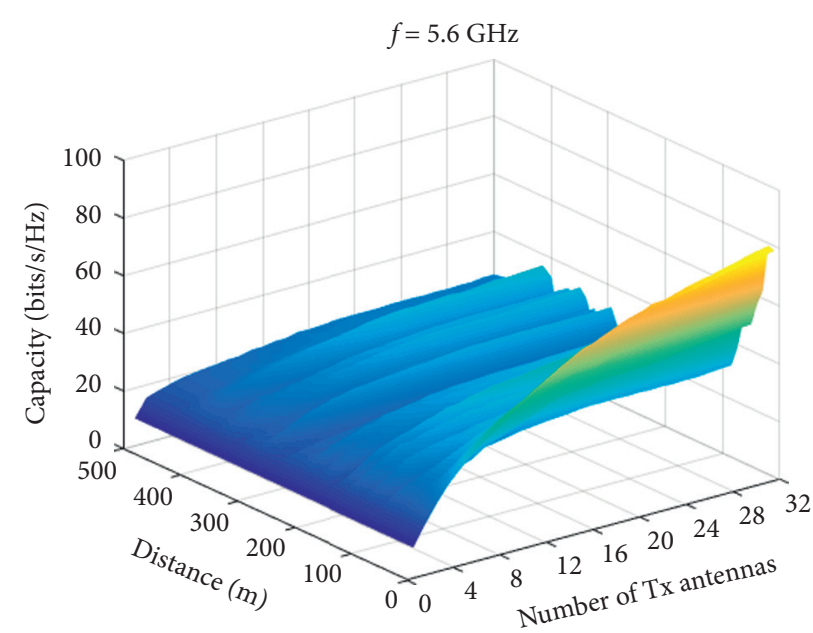

(b)

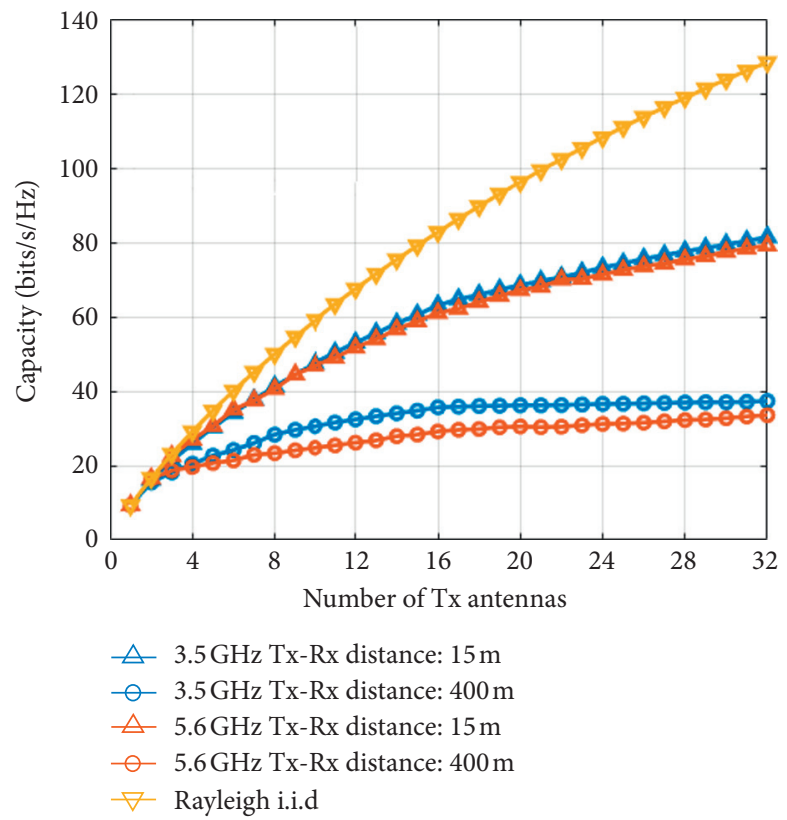

(d)

FIGURE 13: Channel capacity. (a) Channel capacity at $3.5 \mathrm{GHz}$. (b) Channel capacity at $5.6 \mathrm{GHz}$. (c) $32 \times 64$ massive MIMO channel capacity versus distance. (d) Channel capacity versus the number of Tx antenna elements.

Rx. As a reference, the $32 \times 64$ MIMO Rayleigh channel capacity is about $128 \mathrm{bits} / \mathrm{s} / \mathrm{Hz}$. The MIMO Rayleigh channel capacity of $4 \times 4$, which is deployed in the current subway communication system, is about $11 \mathrm{bits} / \mathrm{s} / \mathrm{Hz}$. The channel capacity is considerably lower than the Rayleigh channel capacity under the condition of the same antenna scale. This is because that the tunnel measurement environment in this paper is obviously nonscattering environment, the RMS-DS and RMS-AS are quite small, and the spatial correlation is very large. In addition, the rank reduction of the channel matrix mentioned above also limits the capacity of the channel. However, compared with the $4 \times 4$ MIMO deployment in tunnels, there is still a considerable improvement in channel capacity. The channel capacity of $3.5 \mathrm{GHz}$ is generally greater than $5.6 \mathrm{GHz}$ in the NLOS scenario, which is consistent with the APDP observed in Figure 8. It can be seen that the number and power of multipaths at $3.5 \mathrm{GHz}$ are greater than those at $5.6 \mathrm{GHz}$ in the NLOS area, which results in greater delay spread and angle spread and smaller spatial correlation.

As we all know, under the condition when antennas are not correlated with each other, the channel capacity of MIMO can increase linearly with $\min \left(N_{T}, N_{R}\right)$. It can be seen from Figure 13(d) that at the Tx-Rx distance of $400 \mathrm{~m}$, the channel capacity converges rapidly as the number of $\mathrm{Tx}$ antenna elements increases. When the number of Tx antenna elements is larger than 16, the channel capacity remains relatively constant, and the $3.5 \mathrm{GHz}$ and $5.6 \mathrm{GHz}$ channel capacities are $37 \mathrm{bits} / \mathrm{s} / \mathrm{Hz}$ and $33 \mathrm{bits} / \mathrm{s} / \mathrm{Hz}$, respectively. At the Tx-Rx distance of $15 \mathrm{~m}$, the channel 
capacity at $5.6 \mathrm{GHz}$ and $3.5 \mathrm{GHz}$ is relatively close. When the number of $\mathrm{Tx}$ antenna elements reaches 32, the channel capacity is about $80 \mathrm{bits} / \mathrm{s} / \mathrm{Hz}$.

\section{Conclusion}

In this article, we carried out narrowband measurements at five potential frequency bands, and $32 \times 64$ broadband massive MIMO channel is analyzed in the tunnel scenario of Shanghai Metro Line 7. The tunnel is divided into two propagation areas: the LOS area and the NLOS area. Path loss, PDP, RMS-DS, RMS-AS, channel matrix eigenvalue, and channel capacity have been investigated based on the acquired channel impulse response. The proposed method will have many fruitful applications for the massive MIMO channel-based 5G mobile communications in underground tunnels, mines, and railways. From this article, we can summarize the following conclusions:

(1) The overall path loss exponent in the tunnel is smaller than that of the free space. From a comprehensive comparison of measured frequencies, it is inferred that $5.6 \mathrm{GHz}$ has the smallest path loss attenuation rate in the LOS area, while $10 \mathrm{GHz}$ and $28 \mathrm{GHz}$ have smaller path loss exponents in the NLOS area

(2) The waveguide effect of the tunnel limits the RMSDS and RMS-AS of the channel. The multipath delay is within $100 \mathrm{~ns}$, the maximum value of RMS-DS is less than $15 \mathrm{~ns}$, and the mean value of RMS-AS is less than $6.15^{\circ}$. RMS-DS and RMS-AS attenuate rapidly as the $\mathrm{Tx}-\mathrm{Rx}$ distance increases

(3) In the tunnel scenario, the eigenvalue of the channel matrix decays quickly, and the channel rank reduction is serious. The measured $32 \times 64$ MIMO channel capacity at $3.5 \mathrm{GHz}$ has a maximum value of $85 \mathrm{bits} / \mathrm{s} / \mathrm{Hz}$ and a minimum value of $28 \mathrm{bits} / \mathrm{s} / \mathrm{Hz}$. There is a big gap with the Rayleigh channel due to the high similarity of multipath components. However, compared with the MIMO system deployed in the contemporary communication system in tunnels, the measured channel capacity has been greatly improved. In the NLOS scenario, as the number of Tx antenna elements increases, channel capacity shows a trend of rapid convergence

(4) Through the comparison of the measured frequency bands, RMS-DS, RMS-AS, and channel capacity at $3.5 \mathrm{GHz}$ are slightly larger than these at $5.6 \mathrm{GHz}$

\section{Data Availability}

The measurement data have not been made available because of intellectual property protection. Furthermore, the data are shared with our partner company and will be used in our future work.

\section{Conflicts of Interest}

The authors declare that they have no conflicts of interest.

\section{Acknowledgments}

This work was supported by the National Natural Foundation of China under Grant 61871261 and the Scientific Research Project of Shanghai Shentong Metro Group Co., Ltd. (JS-KY19R031).

\section{References}

[1] M. Lienard, P. Degauque, J. Baudet, and D. Degardin, "Investigation on MIMO channels in subway tunnels," IEEE Journal on Selected Areas in Communications, vol. 21, no. 3, pp. 332-339, 2003.

[2] J. Molina-Garcia-Pardo, M. Lienard, P. Degauque, D. G. Dudley, and L. Juan-Llacer, "Interpretation of MIMO channel characteristics in rectangular tunnels from modal theory," IEEE Transactions on Vehicular Technology, vol. 57, no. 3, pp. 1974-1979, 2008.

[3] J.-M. Molina-Garcia-Pardo, M. Lienard, P. Degauque, C. Garcia-Pardo, and L. Juan-Llacer, "MIMO channel capacity with polarization diversity in arched tunnels," IEEE Antennas and Wireless Propagation Letters, vol. 8, pp. 11861189, 2009.

[4] J. A. Valdesueiro, B. Izquierdo, and J. Romeu, "MIMO channel measurement campaign in subway tunnels," in Proceedings of the Fourth European Conference on Antennas and Propagation (EuCAP), pp. 1-4, Barcelona, Spain, April 2010.

[5] J. A. Valdesueiro, B. Izquierdo, and J. Romeu, "On 2 $\$ \backslash, \mid$ times $\backslash, \$ 2$ MIMO observable capacity in subway tunnels at \$C\$ -band: an experimental approach," IEEE Antennas and Wireless Propagation Letters, vol. 9, pp. 1099-1102, 2010.

[6] D. Li and J. Wang, "Effect of antenna parameters on the field coverage in tunnel environments," International Journal of Antennas and Propagation, vol. 2016, Article ID 8180124, 10 pages, 2016.

[7] R. Sun, D. W. Matolak, C. Tao et al., "Investigation of MIMO channel characteristics in a two-section tunnel at 1.4725 $\mathrm{GHz}$," International Journal of Antennas and Propagation, vol. 2017, Article ID 3693149, 12 pages, 2017.

[8] J. Moreno García-Loygorri, L. De Haro, C. Rodríguez, L. Cuéllar, and J. M. Riera, "Influence of polarization on keyhole probability on a MIMO-OFDM train-to-wayside system on tunnels," IEEE Antennas and Wireless Propagation Letters, vol. 14, pp. 1798-1801, 2015.

[9] J. Moreno, L. de Haro, C. Rodríguez, L. Cuéllar, and J. M. Riera, "Keyhole estimation of an MIMO-OFDM trainto-wayside communication system on subway tunnels," IEEE Antennas and Wireless Propagation Letters, vol. 14, pp. 88-91, 2015.

[10] J. Moreno, J. M. Riera, L. de Haro et al., "MIMO keyholes on tunnels: Measurements," in Proceedings of the European Conference on Antennas and Propagation (EuCAP), pp. 1-3, Davos, Switzerland, April 2016.

[11] J. Li, Y. Zhao, J. Zhang, R. Jiang, C. Tao, and Z. Tan, "Radio channel measurements and analysis at $2.4 / 5 \mathrm{GHz}$ in subway tunnels," China Communications, vol. 12, no. 1, pp. 36-45, 2015.

[12] B. Izquierdo, S. Capdevila, L. Jofre, and J. Romeu, "Evaluation of MIMO capacity in train tunnels," in Proceedings of the IEEE Antennas and Propagation Society International Symposium, pp. 1365-1368, Honolulu, HI, USA, June 2007.

[13] R. He, Z. Zhong, and C. Briso, "broadband channel long delay cluster measurements and analysis at $2.4 \mathrm{GHz}$ in subway 
tunnels," in Proceedings of the IEEE 73rd Vehicular Technology Conference, pp. 1-5, VTC Spring), Budapest, Hungary, May 2011.

[14] E. Masson, Y. Cocheril, M. Berbineau et al., "MIMO channel characterization in subway tunnel for train-to-wayside applications," in Proceedings of the International Conference on ITS Telecommunications, pp. 732-736, Taipei, Taiwan, November 2012 .

[15] T. Zhou, H. Li, R. Sun, Y. Wang, L. Liu, and C. Tao, "Simulation and analysis of propagation characteristics for tunnel train-ground communications at 1.4 and $40 \mathrm{GHz}$," IEEE Access, vol. 7, pp. 105123-105131, 2019.

[16] Q. Wang, B. Ai, R. He et al., "Measurement-based massive MIMO channel characterization in subway station," in Proceedings of the European Conference on Antennas and Propagation (EuCAP), pp. 1-5, London, UK, March 2018.

[17] J. Li, B. Ai, R. He et al., "Channel characterization for massive MIMO in subway station environment at $6 \mathrm{GHz}$ and $11 \mathrm{GHz}$," pp. 1-5, IEEE Vehicular Technology Conference (VTC-Fall), Chicago, IL, USA, 2018.

[18] F. Challita, V. M. Rodrigo-Peñarrocha, L. Rubio et al., "On the contribution of dense multipath components in an intrawagon environment for $5 \mathrm{G} \mathrm{mmW}$ massive MIMO channels," IEEE Antennas and Wireless Propagation Letters, vol. 18, no. 12, pp. 2483-2487, 2019.

[19] K. Guan, Z. Zhong, B. Ai et al., "Complete propagation model in tunnels," IEEE Antennas and Wireless Propagation Letters, vol. 12, pp. 741-744, 2013.

[20] Y. P. Zhang, "Novel model for propagation loss prediction in tunnels," IEEE Transactions on Vehicular Technology, vol. 52, no. 5, pp. 1308-1314, 2003.

[21] P. Bernardi, D. Caratelli, R. Cicchetti, V. Schena, and O. Testa, "A numerical scheme for the solution of the vector parabolic equation governing the radio wave propagation in straight and curved rectangular tunnels," IEEE Transactions on Antennas and Propagation, vol. 57, no. 10, pp. 3249-3257, 2009.

[22] X. Zhang and C. D. Sarris, "Statistical modeling of electromagnetic wave propagation in tunnels with rough walls using the vector parabolic equation method," IEEE Transactions on Antennas and Propagation, vol. 67, no. 4, pp. 2645-2654, 2019.

[23] X. Zhang, N. Sood, and C. D. Sarris, "Fast radio-wave propagation modeling in tunnels with a hybrid vector parabolic equation/waveguide mode theory method," IEEE Transactions on Antennas and Propagation, vol. 66, no. 12, pp. 6540-6551, 2018.

[24] L. Hao, J. Rodríguez-Piñeiro, X. Cai et al., "Measurementbased double-directional polarimetric characterization of outdoor massive MIMO propagation channels at $3.5 \mathrm{GHz}$," in Proceedings of the IEEE International Workshop on Signal Processing Advances in Wireless Communications (SPAWC), pp. 1-5, Atlanta, GA, USA, November 2020.

[25] L. Hao, J. Rodríguez-Piñeiro, X. Yin, and H. Wang, "Measurement-based massive MIMO polarimetric channel characterization in outdoor environment," IEEE Access, vol. 7, pp. 171285-171296, 2019.

[26] A. Goldsmith, Wireless Communications, Cambridge University Press, Cambridge, UK, 2005.

[27] S. K. Kalyankar, Y. H. Lee, and Y. S. Meng, "Two-slope path loss model for curved-tunnel environment with concept of break point," IEEE Transactions on Intelligent Transportation Systems, vol. 21, 2020.

[28] D. P. Gaillot, E. Tanghe, W. Joseph et al., "Polarization properties of specular and dense multipath components in a large industrial Hall," IEEE Transactions on Antennas and Propagation, vol. 63, no. 7, pp. 3219-3228, 2015.

[29] D. Dudley, M. Lienard, S. Mahmoud, and P. Degauque, "Wireless propagation in tunnels," IEEE Antennas and Propagation Magazine, vol. 49, no. 2, pp. 11-26, 2007.

[30] G. J. Foschini and M. J. Gans, "On limits of wireless communications in a fading environment when using multiple antennas," Wireless Personal Communications, vol. 6, no. 3, pp. 311-335, 1998. 